\title{
Single-Ended Transition State Finding with the Growing String Method
}

\author{
Paul M. Zimmerman*
}

Reaction path finding and transition state (TS) searching are important tasks in computational chemistry. Methods that seek to optimize an evenly distributed set of structures to represent a chemical reaction path are known as double-ended string methods. Such methods can be highly reliable because the endpoints of the string are fixed, which effectively lowers the dimensionality of the reaction path search. String methods, however, require that the reactant and product structures are known beforehand, which limits their ability for systematic exploration of reactive steps. In this article, a single-ended growing string method (GSM) is introduced which allows for reaction path searches starting from a single structure. The method works by sequentially adding nodes along coordinates that drive bonds, angles, and/or torsions to a desired reactive outcome. After the string is grown and an approximate reac- tion path through the TS is found, string optimization commences and the exact TS is located along with the reaction path. Fast convergence of the string is achieved through use of internal coordinates and eigenvector optimization schemes combined with Hessian estimates. Comparison to the doubleended GSM shows that single-ended method can be even more computationally efficient than the already rapid doubleended method. Examples, including transition metal reactivity and a systematic, automated search for unknown reactivity, demonstrate the efficacy of the new method. This automated reaction search is able to find 165 reaction paths from 333 searches for the reaction of $\mathrm{NH}_{3} \mathrm{BH}_{3}$ and $(\mathrm{LiH})_{4}$, all without guidance from user intuition. (c) 2015 Wiley Periodicals, Inc.

DOI: $10.1002 /$ jcc.23833

\section{Introduction}

Modern computational chemistry has evolved a number of methods to characterize rates of chemical reaction. Among them, transition state (TS) locating techniques have been used alongside quantum chemical methods to quantify barriers of innumerable elementary reactions and provide immense insight into a wide variety of chemistries. ${ }^{[1-7]}$ Despite this success, most methods for finding TS's still require substantial computational and human effort. Locating TS's, however, is considered worth this effort because TS's contain detailed atomistic descriptions of key mechanistic steps in chemical processes.

While quantum chemistry can provide accurate, $a b$ initio predictions of energetics and gradients in many chemical systems, the relatively high cost of these methods makes TS finding especially challenging. Even when using efficient methods such as density function theory, it becomes costly to perform searches for TS's in realistic model systems with large numbers of atoms. In addition to the computational cost of quantum chemistry, TS finding methods usually must be guided toward a desired TS, a nontrivial task. To succeed, the correct reaction direction must be consistently available, which is a substantial challenge if an accurate approximation of the TS structure is not available. Many methods also rely on the availability of accurate second derivative information to converge to the exact TS. Methods relying on exact Hessians are limited in applicability for larger systems due to the high cost of such computations.

From a user's standpoint, the combination of relatively slow computations with lack of precision knowledge of TS structure leads to a challenging problem. With sufficient experience, skilled users can master specific TS finding techniques but success remains out of reach for less-committed users. Generally speaking, there is a significant need to lower the computational cost of TS finding while also reducing the amount of expert skill and chemical intuition required to successfully locate TS's.

TS finding methods typically fall into one of two categories: single-ended $^{[8-41]}$ and double-ended methods. ${ }^{[42-72]}$ Singleended methods operate on a single chemical structure and attempt to locate a TS by systematically adjusting the structure until it reaches the TS. Double-ended methods require an input of two structures which represent the starting and ending configurations that are connected by a TS. Using this input, a discretized reaction path is constructed where the chain of structures represents an approximate minimum energy path connecting the endpoints. Compared to singleended methods, double-ended methods are typically more reliable and less prone to wandering to undesired regions due to fixing the endpoints of the path. Conversely, the advantage of single-ended methods is that they are able to operate even when the product of the reaction is not known. In many cases, however, single-ended methods are used with a target reaction in mind, reducing this advantage. Single-ended coordinate driving approaches, where specific coordinates are shifted

\footnotetext{
P. M. Zimmerman

Department of Chemistry, University of Michigan, 930 N. University Ave, Ann Arbor, Michigan 48109

E-mail: paulzim@umich.edu

(c) 2015 Wiley Periodicals, Inc.
} 
toward a TS, have been developed with such target reactions in mind. ${ }^{[73-80]}$

The growing string method (GSM) is one technique designed to reduce human and computational effort in locating TS's and reaction paths. ${ }^{[65]}$ By growing structures along a discretized path connecting two intermediates inward toward the TS, the method rapidly forms an accurate approximation to the TS without requiring an initial guess at the TS structure. Further developments have allowed GSM to locate the exact TS along the reaction path without a performing a separate computation or computing the exact Hessian. ${ }^{[72]}$ Despite these advances, GSM can only operate when the product of the chemical step is known and works best when a single elementary step connects the two input intermediates. During growth, GSM can be conceived of as a two-sided driving coordinate method, where coordinates are driven along the GSM reaction tangent direction. This observation presents an interesting opportunity to use driving coordinates in place of the GSM reaction tangent to grow the string, resulting in a singleended string method. Importantly, these driving coordinates can be selected automatically by an algorithm that considers possible chemical rearrangements of the system, resulting in an unguided, predictive search for reactions.

The present article describes a new single-ended GSM, which can locate a reaction path and TS starting from a single structure. Instead of specifying the product structure, the initial conditions of the search are the qualitative characteristics of the intended reaction. The method begins by driving the specified coordinates until a TS region is passed over, then the string is refined until the reaction path and TS are converged. The initial coordinates serve only to drive the string to its destination but are discarded in the subsequent reaction path optimization. This allows some toleration of inaccuracy in choice of reaction coordinate, as low barrier reaction paths roughly parallel to the designated reaction coordinates can be found via string optimization. After describing the method, examples from reactions involving main-group and transition metal reactions will demonstrate the usability of the new approach. Finally, to demonstrate fully automated TS finding, a combinatorial set of reaction coordinates is used to predict previously unknown reactions involving $\mathrm{NH}_{3} \mathrm{BH}_{3}$ and $(\mathrm{LiH})_{4}$. This last example allows single-ended GSM to be operated in a truly predictive fashion, without bias from chemical intuition.

\section{Method}

\section{String method with exact TS search}

The new method uses delocalized internal coordinates ${ }^{[34]}$ constructed from a set of primitive internals (bonds, angles, torsions ${ }^{[71]}$ to describe the string and reaction tangents. Delocalized internals are a nonredundant set that exactly spans the $3 \mathrm{~N}-6$ dimensional space of the molecule. Changes in each node's geometry are described by column vectors of the (rectangular) matrix $U^{(i)}$ which represent a linear combination of primitive internals and superscript (i) refers to the node number. Thus, each $U_{k}^{(i)}$ is a vector representing motion along one orthogonal delocalized internal coordinate. Compared to Cartesian coordinates, delocalized internals accelerate convergence by providing an improved coordinate system for optimization and also greatly improve the description of reaction tangents, leading to higher-quality node addition and less need for subsequent optimization. Importantly, delocalized internal coordinates allow the use of practically any number of primitive internal coordinates because the primitive set can always be transformed into a combination that spans the $3 \mathrm{~N}-6$ degrees of freedom. During string optimization (not growth), the RP tangent, $U_{C}$, between nodes $i$ and $j$ is defined as

$$
U_{C}=\alpha \sum_{k=1}^{3 N-6}\left\langle\Delta q \mid U_{k}^{(i)}\right\rangle U_{k}^{(i)}
$$

with the vector $\Delta q$ being the change in (all) primitive coordinates from node $i$ to node $j$,

$$
\Delta q=q^{(j)}-q^{(i)}
$$

where $q^{(i)}$ are the primitive coordinates for node $i$ and $\alpha$ is a normalization factor. This setup for the reaction tangent allows each node to have a unique delocalized internal coordinate system and handle reaction tangents via projection of changes in primitive internal coordinates $(\Delta q)$ into each coordinate system $U^{(i)}$.

$U_{C}$ is projected out of the remaining internal coordinates $U^{(i)}$ via Gram-Schmidt orthonormalization to form a nonredundant set spanning $3 \mathrm{~N}-7$ degrees of freedom and the final vector becomes $U_{c}$. This vector serves to constrain the nodes from collapsing back to the reactant or product. While the constraint vector $U_{C}$ is usually fixed, steepest ascent steps on the TS node result in a climbing image reaction path search. $^{[49]}$

Optimization of each node is achieved via an eigenvector optimization $^{[34,36,41]}$ strategy, using

$$
\Delta \tilde{U}_{k}=\frac{-\tilde{g}_{k}}{\tilde{H}_{k k}+\lambda}
$$

with $\tilde{U}_{k}$ are the eigenvectors of the Hessian, $\tilde{g}_{k}$ being the gradient in the eigenvector coordinates, $\tilde{H}_{k k}$ are diagonal elements of the diagonalized Hessian, and $\lambda$ is a scaling factor. The Hessians, $H$, are initially created using a diagonal primitive coordinate Hessian, ${ }^{[72]}$ which is converted into the delocalized internal coordinates via

$$
H=U^{T} H^{\text {prim }} U
$$

and updated using BFGS and Bofill update strategies as the optimization proceeds. ${ }^{[81-85]}$

The eigenvector optimization algorithm also allows exact TS searches to be performed in the full $3 \mathrm{~N}-6$ dimensionality. These searches, which initiate after a climbing image search that brings the TS node close to the exact TS, follow the Hessian eigenvector with the highest overlap to the reaction path direction defined by eq. (1). This strategy removes the 
ambiguity in selecting the TS direction while simultaneously enforcing that the TS remains along the reaction path. ${ }^{[72]}$

\section{Driving in internal coordinates}

One limitation of double-ended string methods is that the reactant and product are required as a starting condition. ${ }^{\left[{ }^{85-87]}\right.}$ This fact motivates the creation of a single-ended GSM, where the benefits of double-ended methods (robust convergence, no need for a guess at the TS structure) can be used to find TS's starting from a single structure. In such a method, the starting structure must migrate to a nearby intermediate, preferably one elementary step distant. Once this intermediate is found, the string method can be optimized to find an accurate reaction path as usual. To achieve this, driving coordinates are introduced into GSM.

To operate GSM with driving coordinates, the tangent vector during the growth stage is replaced by

$$
U_{\mathrm{C}}=\alpha \sum_{k=1}^{3 N-6}\left\langle\delta q \mid U_{k}^{(i)}\right\rangle U_{k}^{(i)}
$$

where $\delta q$ is a primitive internal coordinate vector describing the desired reaction direction. $\delta q$ can include any combination of bonds, angles, or torsions. $\delta q$ can use any primitive internal coordinates, including those not present in the reactant structure: additional driving coordinates are simply added to the coordinate system at the beginning of the computation. Coordinates not being driven are set to zero in $\delta q$, which allows them to freely vary during optimization.

The tangent vector $U_{C}$ is a single degree of freedom representing the collective driving coordinates. This means that when a reaction does not occur synchronously along the driving coordinates, the remaining $3 \mathrm{~N}-7$ coordinates are optimized to yield an asynchronous reaction path.

In practice, $\delta q$ is formed by considering the major bond and angle transformations that are characteristic of the desired reaction. For bond formation, elements of $\delta q$ are scaled such that if the primitive bond coordinate of the growth node approaches zero, the corresponding element of $\delta q$ is reduced to zero. This restriction specifically affects the situation where more than one bond addition is driven and one bond forms sooner than the others, preventing interatomic distances from ever approaching zero. For bond dissociations, $\delta q$ is reduced to zero as the bond distance approaches four times the sum of the covalent radii, indicating the bond is already broken. Usually, angle and torsion coordinates are not driven for reactions involving bond breaking and forming, but angles and torsions are updated by optimization as the string grows. For reactions dominated by changes in angles, $\delta q$ is set with the desired direction of reaction: for angles, to increase or decrease the angle, and for torsions, to rotate clockwise or counterclockwise. Once sufficient nodes have been added along $\delta q$ to reach a stable structure, the vectors $\delta q$ are no longer used. This means that once the string reaches its product, the method switches to using the reaction tangents defined in eq. (1).

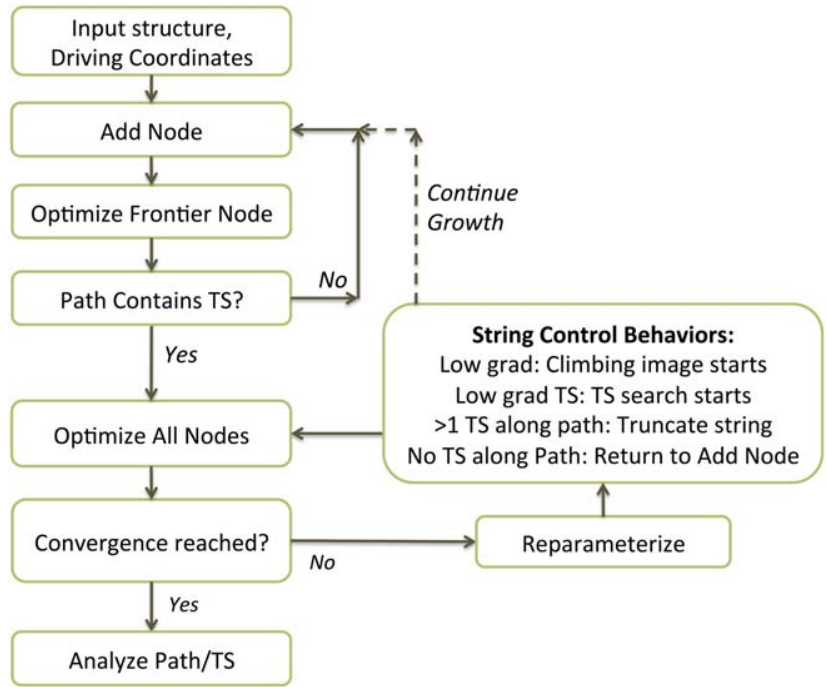

Figure 1. Process flow for single-ended GSM. [Color figure can be viewed in the online issue, which is available at wileyonlinelibrary.com.]

During the growth phase of single-ended GSM, a new node is added along the vector $U_{C}$, and then this node is optimized using $U_{C}$ as a constraint. New frontier nodes can be added and optimized, one at a time, until the reaction path passes over a TS and near a new intermediate. After growth is completed, the string is reparameterized to maintain equal node spacing and $U_{C}$ from eq. (1) is used to represent the reaction path instead of the driving coordinates. Importantly, this means that the single-ended and double-ended GSM's can give the same reaction path if the single-ended method grows to the same product structure as the double-ended method.

\section{Single-ended GSM}

Combining GSM with driving coordinates yields the starting point for a single-ended GSM. The overall scheme for this method is shown in Figure 1. Similar to double-ended GSM, growth and optimization steps are cycled until the string connects reactant to product. The vital difference is that, during the growth phase, nodes are only added from the reactant side and the constraint $U_{C}$ is given by eq. (5) instead of eq. (1). Unlike GSM, where the product is known, single-ended GSM must monitor for a TS to appear along the path to know whether to continue growing. Two criteria are used to evaluate this, either (1) the frontier node is lower in energy than the previous node (by a threshold value), or (2) the frontier node's constraint gradient is positive. Both criteria indicate the string has passed into a new intermediate basin. Once the frontier node is over the TS, additional nodes are added and optimized to complete the string. Instead of enforcing that the final node be fully optimized, two nodes are added following the TS to maintain low numbers of nodes, and thus, low computational cost. Following TS convergence, the final node is optimized to the reaction product.

After growth is completed, two conditions are monitored during the optimization phase to ensure that the reaction path contains one, and only one, TS along the path. If more than one TS 
is detected, the string is truncated to the first detected intermediate and optimization continues. If no TS is detected, the string returns to the growth phase until a TS is found. In principle "perfect optimization" during growth-consisting of full node optimization with a constraint vector that is the exact tangent to the desired reaction path-would avoid both of these conditions. In practice, however, the constraint vector is not an exact representation of the reaction path, so situations where multiple or no TS's appear must be handled accordingly.

\section{Operating the single-ended GSM}

Where the double-ended GSM required two input structures and the number of nodes, single-ended GSM requires one initial structure and a set of driving coordinates, where the latter can be determined automatically. The number of nodes for the singleended string is not set via input but actively determined while the string is growing. To adequately space the nodes during new node addition, the maximum shift along the constraint coordinate is fixed (the minimum value linearly scales with the length of vector $\delta q$ ). This value was fixed to $0.8 \AA$-radians and appears to work well for numerous test cases. Only the frontier node is optimized during the growth phase of single-ended GSM.

After the growth phase and initial reparameterization of the string, the method cycles through eigenvector optimization of all nodes (including Hessian updates), convergence checks, and reparameterization steps (Fig. 1). Three optimization steps are taken per node prior to each reparameterization, and six optimization steps are taken on the TS node once the TS search begins. When the total gradient falls low enough, climbing image starts and later the eigenvector following TS search begins. The run finishes when the TS node is converged to a tight gradient threshold.

\section{Approximating the TS eigenvector}

The present TS optimization algorithm relies on approximate Hessian information to guide the eigenvector following routine. While exact Hessians would in principle be valuable to guide the TS search, in practice these computations are too expensive to readily employ for large systems. For all nodes, the initial Hessians are constructed from a diagonal primitive Hessian [eq. (4)] and updated as the optimization proceeds. A curvature approximation ${ }^{[88]}$ based on neighboring nodes is used to estimate the TS eigenvector,

$$
C=\frac{2 E_{\mathrm{TS}-1}}{a(a+b)}-\frac{2 E_{\mathrm{TS}}}{a b}+\frac{2 E_{\mathrm{TS}+1}}{b(a+b)}
$$

where $a$ and $b$ are the distances between the nodes prior to and following the TS, respectively. This curvature is projected into the approximate Hessian via

$$
\Delta H=\left(C-U_{C}^{\top} H U_{C}\right) U_{C} U_{C}^{\top}
$$

which results in a single negative eigenvalue if the Hessian was previously positive definite (which is enforced by the BFGS Hessian update ${ }^{[81-84]}$ ).
At this point, an approximate Hessian with the desired eigenvalue structure is known, but the exact eigenvectors of the exact Hessian are not. Following Head-Gordon, ${ }^{[89]}$ we use a Davidson method to accurately calculate the lowest exact Hessian eigenvectors and eigenvalues when initiating the exact TS search, without needing to compute the exact Hessian. The Davidson method selectively diagonalizes the lowest few eigenvalues of a given (in this case, unknown) matrix, $H_{\text {exact }}{ }^{[90]}$ The initial vectors are taken as the lowest eigenvectors of the approximate Hessian at the TS node after applying eqs. (6) and (7). While the original Davidson method required the exact Hessian matrix, the finite difference formula

$$
H_{\text {exact }} b_{1} \approx \frac{g\left(x+\varepsilon b_{1}\right)-g\left(x-\varepsilon b_{1}\right)}{2 \varepsilon}
$$

avoids the computation of this matrix by substituting gradient information. In (8), $H$ is the (unknown) exact Hessian matrix, $x$ is the current position, $b_{1}$ is a Davidson expansion vector, $g$ is the gradient, and $\varepsilon$ is a small parameter. We refer the reader to Ref. [90] for full details of this procedure. The significant difference with the present work is that delocalized internals are used instead of Cartesian coordinates. After converging the Davidson routine, the resulting eigenvectors are projected into the approximate Hessian [similar to eq. (7)] for TS node optimization.

\section{Computational Details}

The B3LYP density functional ${ }^{[91-93]}$ is utilized for the examples herein, though the method is in no way constrained to this level of theory. The double zeta, polarized $6-31 \mathrm{G}^{* *}$ basis set is used throughout, except transition metals employ the LANL2DZ basis. ${ }^{[94-96]}$ The string method is implemented in $\mathrm{C}++$ and invokes $\mathrm{Q}-$ Chem $4.1^{[97]}$ to provide the quantum mechanical gradients. Images of intermediates were generated in visual molecular dynamics (VMD). ${ }^{[98]}$

A initial maximum optimization step size of 0.1 is applied for all test cases. The eigenvalue shift parameter $\lambda$ of the eigenvector algorithm (see Ref. [72]) is chosen such that the shifted eigenvalues are all positive, except for the exact TS search, where $\lambda$ shifts only the non-TS eigenvalues. During growth, new nodes are optimized until the root mean square (RMS) gradient is below 0.005 Hartree/A, or 30 steps. Convergence of the reaction path is considered complete when the TS node has a small RMS gradient $(<0.0005$ Hartree/A $)$. The climbing image search is initiated after the sum of the perpendicular gradient magnitudes over all nodes, $F$, is converged to $F<0.3$. The exact TS search is initiated when one the three sets of conditions is met: (1) the total gradient is $<0.2$, the TS node is converged to within 10 times the nodal convergence tolerance, and the constraint force is $<0.01$, (2) the total gradient is $<0.1$, the TS node is converged to within 10 times the convergence tolerance and the constraint force is $<0.02$, or ( 3 ) the TS node is within five times the convergence tolerance. The Davidson method is used to construct the two lowest curvature eigenvectors to start the TS search. 


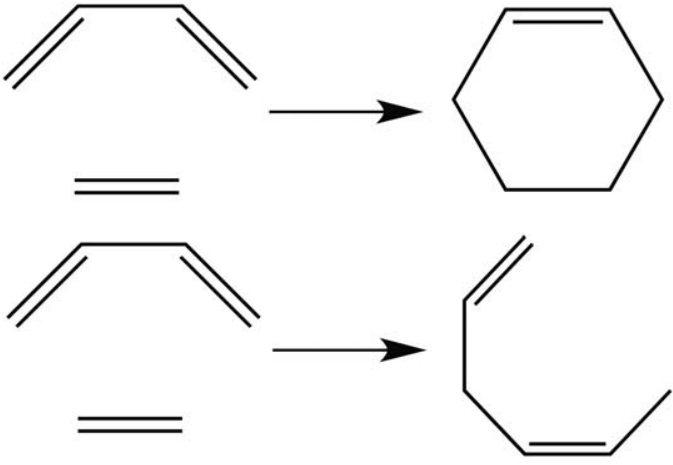

Figure 2. Diels-Alder and Ene reactions.

\section{Results and Discussion}

\section{Comparison of double-ended and single-ended GSM}

To demonstrate the new method, we first compare it to the double-ended version of GSM from Ref. [72]. Like single-ended GSM, double-ended GSM uses a eigenvector optimizer, which was shown to be significantly faster than conjugate gradient optimization. ${ }^{[72]}$ The Davidson algorithm is not available in double-ended GSM, so the curvature at the TS is initially approximated by eq. (5). To draw out differences in the two approaches, three reactions are analyzed using each method.

The first, the Diels-Alder reaction of cis-butadiene with ethylene, ${ }^{[99]}$ is a prototypical cycloaddition involving formation of two $\mathrm{C}-\mathrm{C}$ connections (Fig. 2). Using driving coordinates corresponding to two C-C bond formations, single-ended GSM produces a string consisting of seven nodes and converges to the expected TS at $19.6 \mathrm{kcal} / \mathrm{mol}$. Although additional bond and angle coordinates change over the course of the reaction, specifying only the dominate coordinates was sufficient to drive the string to the desired product. Convergence was reached after 264 gradient computations: out of this total, 78 gradients are needed for the growth phase and 38 for the Davidson procedure. In comparison, double-ended GSM uses 435 gradients to converge using 11 nodes, where only 42 gradients are needed for growth. Overall, both methods reach the same TS, but double-ended GSM requires significantly more gradient computations due to using a larger number of nodes. Using only seven nodes, double-ended GSM is able to converge the TS in only 225 gradients. This latter observation can be explained by double-ended GSM's improved tangent direction during growth, where angles and torsions are included beyond the major bond-making coordinates of single-ended GSM. Double-ended GSM, therefore, anticipates more of the

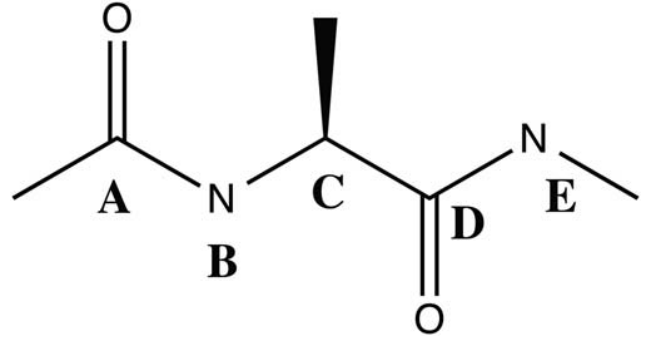

Figure 3. Alanine dipeptide isomerization involving rotation around A-B-C$\mathrm{D}$ and $\mathrm{B}-\mathrm{C}-\mathrm{D}-\mathrm{E}$ dihedral angles.

reaction path, and this information allows the string to grow with higher accuracy, leading to faster convergence.

The choice in number of nodes for double-ended GSM was based on benchmarks from a large set of reactions, where a fixed node number is desirable to minimize the number of variable input parameters. Reducing the number of nodes for double-ended GSM would require tailoring the node number by hand for each reaction, and therefore, single-ended GSM's ability to dynamically choose a number of nodes is advantageous. In single-ended GSM, the final node spacing along the constraint vector $U_{C}$ is on average 1.47 , where double-ended GSM has spacing of 1.05 with 11 nodes. While it was shown that many cases failed to converge using double-ended GSM and only seven nodes, ${ }^{[72]}$ the larger spacing was sufficient to converge the reaction path in this specific case due to the simplicity of the reaction.

The Ene reaction begins from the same substrates as the Diels-Alder (Fig. 2) and is the second case used for comparing the two GSM methods. This reaction consists of breaking an ethylene $\mathrm{C}-\mathrm{H}$ bond along with concerted formation of $\mathrm{C}-\mathrm{C}$ and $\mathrm{C}-\mathrm{H}$ bonds with butadiene in a six-membered ring TS. These three driving coordinates result in single-ended GSM growing to seven nodes after 77 gradient computations and 320 total gradients to converge the TS with a barrier of 41.2 $\mathrm{kcal} / \mathrm{mol}$. In comparison, double-ended GSM with 11 nodes required 365 gradients to converge the TS. Using seven nodes, the double-ended method is able to converge in 251 gradients, which suggests that the improved initial reaction tangent of double-ended GSM results in some speedup compared to single-ended GSM.

As a third comparison, alanine dipeptide conformational isomerization $^{[100]}$ around the two dihedral angles shown in Figure 3 is investigated. This particular case has been shown to be best treated in internal coordinates because Cartesian coordinates tend to cause atoms to collide over the

Table 1. Comparison of single-ended and double-ended growing string methods. 11 nodes are used for double-ended GSM

\begin{tabular}{|c|c|c|c|c|c|c|}
\hline \multirow[b]{2}{*}{ Reaction } & \multirow[b]{2}{*}{ Atoms } & \multirow[b]{2}{*}{ Driving coordinates } & \multicolumn{2}{|c|}{ \# Gradients total } & \multicolumn{2}{|c|}{ \# Gradients growth stage } \\
\hline & & & SE-GSM & DE-GSM & SE-GSM & DE-GSM \\
\hline Diels-Alder & 16 & $C-C$ formation $(\times 2)$ & 264 & 435 & 78 & 42 \\
\hline Ene & 16 & $\begin{array}{c}\mathrm{C}-\mathrm{H} \text { dissociation } \\
\mathrm{C}-\mathrm{H}, \mathrm{C}-\mathrm{C} \text { formations }\end{array}$ & 320 & 365 & 77 & 50 \\
\hline Alanine dipeptide & 22 & Dihedral angles & 165 & 114 & 62 & 42 \\
\hline
\end{tabular}



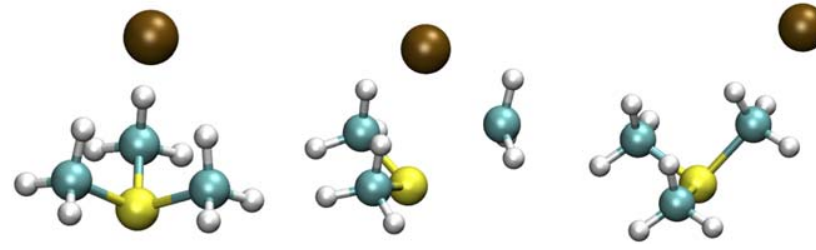

Figure 4. Reaction of $\mathrm{Cl}^{-}$with $\mathrm{S}(\mathrm{CH} 3)_{3}^{+}$a) Reactant complex. b) $\mathrm{S}_{\mathrm{N}} 1$ transition state, front-side attack. c) $\mathrm{S}_{\mathrm{N}} 2$ transition state, backside attack.

isomerization. ${ }^{[71]}$ Single-ended GSM converges to the desired TS at $6.8 \mathrm{kcal} / \mathrm{mol}$ in 165 gradients, using 62 gradients for complete growth to eight total nodes. Using double-ended GSM, 11 nodes converge to the TS using just 114 gradients, 42 of which are required for the growth phase.

For these three test cases, single-ended GSM performs well (see Table 1) compared to double-ended GSM despite that the latter has a distinct advantage: full, a priori knowledge of the product structure. Single-ended GSM is able to achieve convergence in low numbers of gradient calls due to its adaptive number of nodes, where the (small) number of nodes required for consistent description of the reaction path is automatically determined.

\section{Aligning reactions: $\mathrm{S}_{\mathrm{N}} 2$ and $\mathrm{S}_{\mathrm{N}} 1$ examples}

An important advantage of single-ended GSM is that multiple reaction paths can be systematically located by variation of driving coordinates. For instance, reactions might proceed via $\mathrm{S}_{\mathrm{N}} 2$ or $\mathrm{S}_{\mathrm{N}} 1$ type mechanisms, resulting in the same regioconnectivity but not necessarily the same stereoisomer. In general for an $\mathrm{S}_{\mathrm{N}} 2$ reaction, the nucleophile is not always aligned opposite the leaving group in the reactant structure. For instance, in the reaction of trimethylsulfonium chloride, $\mathrm{S}(\mathrm{CH} 3)_{3}^{+} \mathrm{Cl}^{-}$, the outcome of the TS search depends on the approach of $\mathrm{Cl}$ to the $\mathrm{C}$ atom. ${ }^{[75]}$ Therefore, an $\mathrm{S}_{\mathrm{N}} 1$ TS is the closest route of reaction for complexes such as the one shown in Figure $4 a$, where the $\mathrm{Cl}^{-}$is positioned for front-side attack. The mechanism for this rearrangement, however, may be better described by an $\mathrm{S}_{\mathrm{N}} 2$ reaction, which has a barrier more than $10 \mathrm{kcal} / \mathrm{mol}$ lower than $\mathrm{S}_{\mathrm{N}} 1$ in the gas phase. To obtain the $\mathrm{S}_{\mathrm{N}} 2$ reaction, either the driving coordinate must involve angular degrees of freedom, or the reactant complex realigned for backside attack.

The new string method can efficiently capture $\mathrm{S}_{\mathrm{N}} 1$ and $\mathrm{S}_{\mathrm{N}} 2$ reactions when appropriate driving coordinates are selected. In both cases, starting from the structure shown in Figure 4a, the $\mathrm{Cl}-\mathrm{C}$ distance was driven to form a new bond and the $\mathrm{S}-\mathrm{C}$ distance pushed to detachment. Because the $\mathrm{Cl}$ is positioned for front-side attack, the string method captures the $\mathrm{S}_{\mathrm{N}} 1 \mathrm{TS}$ with predissociation of the methyl group. To obtain the $\mathrm{S}_{\mathrm{N}} 2 \mathrm{TS}$, one addition coordinate is added: the $\mathrm{S}-\mathrm{C}-\mathrm{Cl}$ angle, which is set to a target of $180^{\circ}$. In this case, the $S_{N} 2$ TS is found as the $\mathrm{S}-\mathrm{C}-\mathrm{Cl}$ angle is aligned for backside attack by the additional driving coordinate.

In this example, the $S_{N} 1$ reaction path is described by only four nodes and requires 91 gradients to converge the TS. The $\mathrm{S}_{\mathrm{N}} 2$ path required five nodes and 150 gradients for convergence. After converging the TS's, the Davidson procedure was utilized on each reaction paths' TS to determine the curvature of the three lowest Hessian eigenvectors. A single negative eigenvalue for each TS was found, confirming the saddle points. Importantly, this procedure required 46 and 18 gradients for $\mathrm{S}_{\mathrm{N}} 1$ and $\mathrm{S}_{\mathrm{N}} 2$ TS's, respectively, which is relatively inexpensive compared to full Hessian computations.

\section{Transition metal reactivity}

To evaluate the efficacy of the new method for transition metal reactivity, six test cases were developed. These are described in Table 2 and cover polymerizations of olefin and thiophene, $\mathrm{C}-\mathrm{H}$ activation of alkanes and aromatics, and dihydrogen formation. The reactions were inspired by current efforts in transition metal catalysis to activate and transform a wide variety of chemical functionalities.

The first reaction in this set corresponds to the association of methane to trans- $\mathrm{PtCl}_{2}\left(\mathrm{H}_{2} \mathrm{O}\right)_{2}$, a $\mathrm{C}-\mathrm{H}$ activating species. ${ }^{[101-103]}$ The methane-Pt $\sigma$-complex is thermodynamically uphill from the starting species due to the weak electron donation properties of the methane $\mathrm{C}-\mathrm{H}$ bond. Therefore,

Table 2. Transition metal reactions studied using single-ended Growing String

\begin{tabular}{|c|c|c|c|c|}
\hline Reaction & Atoms & Driving coordinates & Gradients & $E_{\mathrm{a}}$ \\
\hline $\mathrm{CH}_{4}$ sigma complex formation at $\mathrm{PtCl}_{2}\left(\mathrm{H}_{2} \mathrm{O}\right)_{2}$ & 14 & $\begin{array}{l}\text { C-Pt formation } \\
\mathrm{H}-\mathrm{Pt} \text { formation }\end{array}$ & 176 & 31.0 \\
\hline Reductive elimination of dithiophene at Ni(diimine) & 25 & $\begin{array}{l}\mathrm{C} 1-\mathrm{C} 2 \text { formation } \\
\mathrm{Ni}-\mathrm{C} 3 \text { formation } \\
\mathrm{Ni}-\mathrm{C} 2 \text { dissociation }\end{array}$ & 278 & 25.1 \\
\hline $\operatorname{Ir}($ pincer $)(\mathrm{H})_{4} \rightarrow \operatorname{Ir}($ pincer $)(\mathrm{H})_{2} \mathrm{H}_{2}$ & 26 & $\begin{array}{l}\mathrm{H}-\mathrm{H} \text { bond formation } \\
\mathrm{I}-\mathrm{H} \text { dissociation }(\mathrm{x} 2)\end{array}$ & 148 & 3.3 \\
\hline $\operatorname{Ir}($ pincer $)(\mathrm{H})_{2}$ Ethane $\mathrm{C}-\mathrm{H}$ activation & 32 & $\begin{array}{c}\mathrm{C}-\mathrm{H} \text { dissociation } \\
\mathrm{C}-\mathrm{Ir} \text { formation } \\
\mathrm{H}-\mathrm{Ir} \text { formation }\end{array}$ & 208 & 22.6 \\
\hline $\mathrm{Ni}$ (diimine) $\mathrm{CH}_{3}$ Ethylene insertion & 19 & $\begin{array}{c}\mathrm{C}-\mathrm{C} \text { formation } \\
\mathrm{Ni}-\mathrm{C} \text { dissociation }(\times 2)\end{array}$ & 285 & 15.8 \\
\hline $\mathrm{Pd}\left(\mathrm{PH}_{3}\right)_{2}$ acetate $\mathrm{C}-\mathrm{H}$ Activation of benzene & 28 & $\begin{array}{l}\mathrm{O}-\mathrm{H} \text { formation } \\
\mathrm{C}-\mathrm{Pd} \text { dissociation } \\
\mathrm{C}-\mathrm{H} \text { dissociation }\end{array}$ & 359 & 10.7 \\
\hline
\end{tabular}



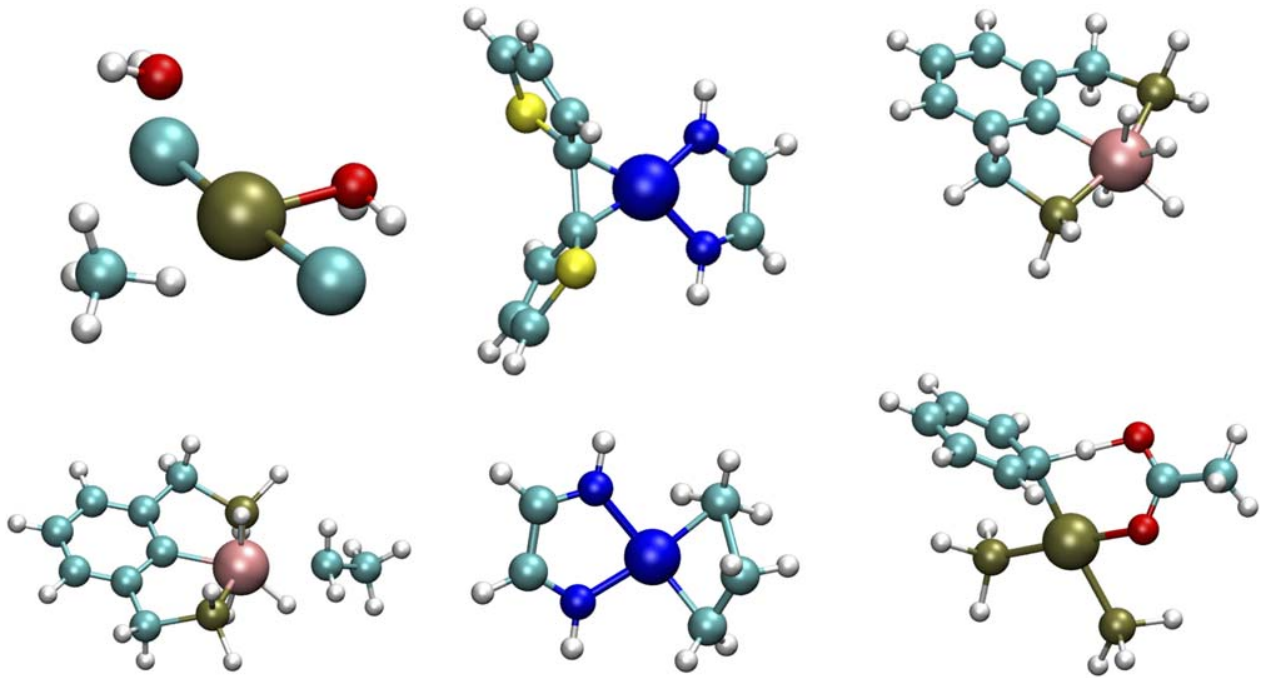

Figure 5. Transition states found using single-ended GSM for transition metal reactions. From upper left, moving left to right: methane association to $\mathrm{PtCl}_{2}\left(\mathrm{H}_{2} \mathrm{O}\right)_{2}$, dithiophene elimination at Ni(diimine), $\mathrm{H}_{2}$ formation at $\operatorname{Ir}$ (pincer), ethane activation at $\operatorname{Ir}($ pincer), ethylene insertion into $\mathrm{Ni}($ diimine)- $\mathrm{CH}$, and $\mathrm{C}-\mathrm{H}$ activation of benzene by $\mathrm{Pd}$-acetate.

displacing $\mathrm{H}_{2} \mathrm{O}$ has a significant barrier that must be accounted for in the process of methane activation. To determine the reaction path, $\mathrm{H}-\mathrm{Pt}$ and $\mathrm{C}-\mathrm{Pt}$ associations were set as the driving coordinates. Using these settings, the string grew to reach the $\sigma$-complex with $\mathrm{H}_{2} \mathrm{O}$ dissociated. After several optimization iterations, the string of seven nodes is able to converge to the expected TS (see Fig. 5, top left). The total cost of the run was 176 gradients to determine the reaction path and barrier of $31.0 \mathrm{kcal} / \mathrm{mol}$.

The second reaction, reductive elimination of dithiophene from $\mathrm{Ni}$ (diimine)(thiophene) ${ }_{2}$, starts with two $\mathrm{sp}^{3} \mathrm{Ni}-\mathrm{C}$ linkages and ends with a new $\mathrm{C}-\mathrm{C}$ bond and a $\mathrm{Ni}-\pi$ complex. This reaction is key to the living polymerization of polythiophene, a semiconducting electronic material. ${ }^{[104,105]}$ To drive this reaction, the $\mathrm{C}-\mathrm{C}$ bond is added with dissociation of one of the two $\mathrm{C}-\mathrm{Ni}$ bonds. Additionally, because the final product is an $\eta^{2} \mathrm{Ni} \pi$-complex, a second $\mathrm{C}-\mathrm{Ni}$ bond is added to account for this feature. This reaction converges after 278 total gradients, resulting in a TS barrier of $25.1 \mathrm{kcal} / \mathrm{mol}$.

For the next reaction, an Ir pincer complex with four hydrogen atoms attached-a key intermediate ${ }^{[106]}$ in catalytic dehydrogenation of ammonia-borane and alkanes-is investigated. This species starts from four single $\mathrm{I}-\mathrm{H}$ bonds and proceeds to form an $\mathrm{H}-\mathrm{H}$ bond while the other two $\mathrm{Ir}-\mathrm{H}$ remain intact. This reaction will be the first step in elimination of $\mathrm{H}_{2}$ from Ir, opening up additional coordination sites for further reaction.

Hypothesizing that the reaction will proceed through concerted $\mathrm{H}-\mathrm{H}$ bond formation and $\mathrm{H}_{2}$ dissociation, three driving coordinates were chosen: $\mathrm{H}-\mathrm{H}$ formation, and two $\mathrm{Ir}-\mathrm{H}$ dissociations. This reaction, however, turned out to not be concerted, but the reaction proceeds stepwise starting with $\mathrm{H}-\mathrm{H}$ formation. This reactive outcome, therefore, is the same as if the reaction hypothesis had been correct: driving just the $\mathrm{H}-\mathrm{H}$ bond formation results in the same TS. Overall, using the first hypothesis resulted in 148 gradient computations being required, where the second converged faster, using only 64 gradients. The major difference in number of optimization iterations can be attributed to the 14 nodes required for the first case, and only five for the second. This result suggests input of accurate reaction coordinates can enhance convergence efficiency but that less accurate reaction coordinates can lead to the same outcome.

The same Ir pincer complex is used in the fourth reaction, $\mathrm{C}-\mathrm{H}$ activation of ethane. In this case, the starting structure is an ethane coordinated to the Ir center, where the latter is octahedral with two $\mathrm{I}-\mathrm{H}$ bonds. $\mathrm{C}-\mathrm{Ir}$ and $\mathrm{H}-\mathrm{Ir}$ bonds are added along with $\mathrm{C}-\mathrm{H}$ dissociation for the driving coordinates. After 208 gradients, the string of seven nodes reaches convergence and results in the expected $\mathrm{C}-\mathrm{H}$ activation reaction shown in Figure 5.

The $\mathrm{Ni}$ diimine species from the dithiophene reductive elimination was chosen to examine a common polymerization reaction: insertion of ethylene into a $\mathrm{Ni}$-methyl bond. ${ }^{[107,108]}$ The $\mathrm{Ni}$-methyl $\mathrm{C}$ bond and one $\mathrm{Ni}$-ethylene $\mathrm{C}$ bond are driven to break along with addition of a $\mathrm{C}-\mathrm{C}$ bond consisting of these two $C$ atoms. This reaction, involving a cationic species, completes without incident using six total nodes and requiring 285 gradients. The barrier for this process is 15.8 $\mathrm{kcal} / \mathrm{mol}$.

The final example consists of a Pd-acetate catalyzed activation of a benzene $\mathrm{C}-\mathrm{H}$ bond. ${ }^{[109-111]}$ The starting species is a cationic $\mathrm{Pd}\left(\mathrm{PH}_{3}\right)_{2}\left(\mathrm{CH}_{3} \mathrm{COO}\right)$ center associated to a benzene via an $\eta^{2} \pi$-complex. Besides breaking the $\mathrm{C}-\mathrm{H}$ bond and adding a $\mathrm{H}-\mathrm{O}$ bond to the acetate, the two $\mathrm{C}-\mathrm{Pd}$ bonds were also included in the coordinate driving. The first C-Pd bond was added to ensure this connection stayed in place, while the second C-Pd was dissociated such that the product would contain a single benzyl $C$ to Pd bond. After 359 gradients, the string of six nodes converges to a concerted TS with a barrier of $10.7 \mathrm{kcal} / \mathrm{mol}$.

Overall, single-ended GSM is able to handle a variety of transition metal catalyzed reactions reliably and accurately. Table 2 

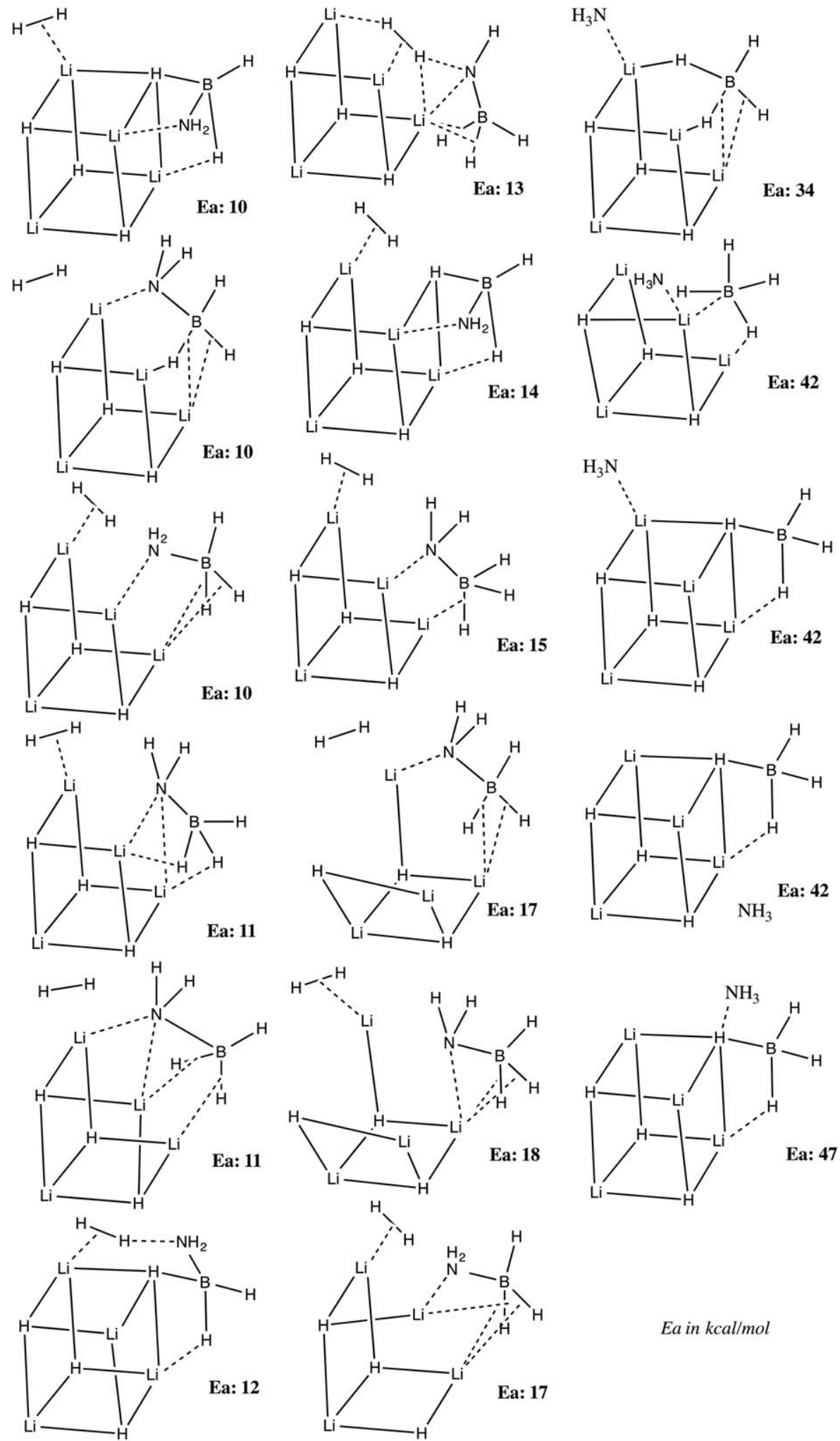

Figure 6. Reaction products discovered using combinatorial reactive hypothesis generation and single-ended GSM. Only pathways with barriers lower than $50 \mathrm{kcal} / \mathrm{mol}$ are shown.

lists the number of gradients required for these reactions. The reactions converged using less than 400 gradients each, where on average 242 gradients were required. In each case, the
Davidson procedure confirmed the presence of a single negative eigenvalue of the Hessian corresponding to the TS motion. 


\section{Automated and predictive single-ended reaction searching}

Single-ended GSM can also be used without manually specifying the initial reaction coordinates, thus, making the method fully predictive of elementary reaction steps without using chemical intuition. To demonstrate how this can be achieved, the reaction of ammonia-borane $\left(\mathrm{NH}_{3} \mathrm{BH}_{3}\right)$ and a lithium hydride nano crystal $(\mathrm{LiH})_{4}$ was investigated. This reaction has been shown to be a promising method for generation of a new hydrogen storage material, $\mathrm{LiNH}_{2} \mathrm{BH}_{3}{ }^{[112]}$ which has attracted some interest due to its relative ease of $\mathrm{H}_{2}$ removal compared to ammonia-borane. ${ }^{[13]}$

Because this reactivity is not well understood, a variety of reactive hypotheses could be made involving formation of $\mathrm{H}_{2}$, bridged $\mathrm{Li}-\mathrm{H}-\mathrm{B}$, and $\mathrm{Li}-\mathrm{N}$ type structures as well as crystal decomposition. However, the great number of potential reactions involved makes manual investigation of the many reaction combinations (which may occur individually or in concerted steps) a daunting task. To survey the reactive landscape, a combinatorial set of reactive coordinates ${ }^{[85,87]}$ were chosen to be used as input driving coordinates for single-ended GSM. This set consists of combinations where up to two atomic connections were formed and two broken, and angle coordinates were not explicitly sampled. To reduce the search space to a reasonable scope, specific atoms were included in this reactive set, including $\mathrm{N}$ and $\mathrm{B}$ of ammonia-borane and two $\mathrm{H}$ connected to this molecule. Two $\mathrm{Li}$ and two $\mathrm{H}$ of the lithium hydride cluster were also allowed to be included in the reactive set. Other atoms were included in the simulation but were not included in the combinatorial set of driving coordinates.

In total, 333 sets of reactive coordinates were made using this combinatorial technique. Out of this total, 165 meaningful reaction paths were found. The remaining GSM runs followed poor reactive coordinates, for instance leading to formation of new connections that were energetically unstable that were difficult to optimize. Figure 6 summarizes the reactive steps with barriers lower than $50 \mathrm{kcal} / \mathrm{mol}$, which consists of 17 unique reactions. By unique, we refer specifically to qualitatively different connectivity of the reaction products. Many of these 17 reactions were found more than once, indicating the approximate reaction directions will proceed to low energy paths upon string optimization. For instance, the $\mathrm{H}_{2}$ formation reaction with activation barrier of $13 \mathrm{kcal} / \mathrm{mol}$ (top center of Fig. 6) was found 11 times. The $\mathrm{H}_{2}$ formation reaction with barrier $14 \mathrm{kcal} / \mathrm{mol}$ (directly below the previously mentioned reaction) was located 12 times. Four of the 17 reactions were found only once, where on average each was found 4.6 times. This indicates that similar sets of driving coordinates will lead to similar outcomes, making single-ended GSM relatively insensitive to coordinate choice in many cases.

Out of the great variety of located reactions, the most feasible involve $\mathrm{H}_{2}$ elimination through $\mathrm{N}-\mathrm{H}$ hydrogen transfer or $\mathrm{BH}_{3}$ attachment to the $\mathrm{LiH}$ structure, resulting in $\mathrm{Li}-\mathrm{H}-\mathrm{B}$ bridges. Overall, because reactive steps with barriers lower than $20 \mathrm{kcal} / \mathrm{mol}$ should proceed efficiently at room temperature, the reaction discovery procedure resulted in 12 highly plausible elementary reactions.

\section{Conclusions}

Locating TS's is a vital task in computational chemistry, so extensive efforts are being spent on developing new methods for application to a variety of chemical reactions. The proposed single-ended GSM adds to this repertoire of TS-finding techniques by bridging single- and double-ended methods in a single tool. By doing so, limitations of each class of method can be reduced. For instance, while single-ended methods can be started with a single structure, these do not guarantee that the located TS is the desired TS or that it connects to the starting structure. Conversely, while double-ended methods are highly reliable and do not share this problem, these must be started from a reactant-product pair. In many cases, the product structure is not necessarily already available. Singleended GSM avoids these issues by moving from the reactant structure to a nearby intermediate, which allows an accurate reaction path and TS to be found simultaneously. Furthermore, the new method simplifies TS finding by combining three computations that might otherwise be separate: determining the product, reaction path optimization, and exact TS search.

Single-ended GSM has a wealth of capabilities that are only found piecewise in existing methods. While exact TS searching is nothing new, the new method has advantages over singleended TS finders like the dimer method because it locates the TS, reaction product and reaction path in a single computation. Furthermore, single-ended GSM's use of internal coordinates allows a systematic combination of bond-making and bond-breaking coordinates to drive reaction outcomes; the dimer method relies on random moves away from the reactant structure, making its TS searches less systematic and more reliant on chance. Single-ended GSM maintains the high efficiency and accuracy of double-ended methods but requires less user effort and intuition because the product does not need to be specified before starting the search. Overall, this gives a unique place for single-ended GSM: it surpasses existing methods in many metrics including ease of use and cost, while also providing new capabilities that have not before been demonstrated such as systematic reaction searching along chemical bonding coordinates.

In benchmarks shown in this article, between 64 and 359 gradients were required to form a reaction path and converge the exact TS. The cost of single-ended GSM is relatively low for two reasons: (1) only gradient computations are required, and (2) a relatively small number of nodes-determined adaptively for each reaction-is necessary to discretize the reaction path. Overall, this means the method will be highly useable for quantum chemical applications, where the cost of gradient computations is relatively high. The low degree of user effort required to operate single-ended GSM means that novice and expert users should find this technique highly practical. Finally, the single-ended GSM will also see continued use by systematic sampling of reaction coordinates, such as was achieved in the ammonia-borane lithium hydride reactivity, where many feasible reactions were predicted without human guidance.

Keywords: transition state $\cdot$ single-ended $\cdot$ string method 
How to cite this article: P. M. Zimmerman J. Comput. Chem. 2015, 36, 601-611. DOI: 10.1002/jcc.23833

[1] A. T. Ziegler, Chem. Rev. 1991, 91, 651.

[2] W. Kohn, A. D. Becke, R. G. Parr, J. Phys. Chem. 1996, 100, 12974.

[3] R. G. Parr, W. Yang, J. Am. Chem. Soc. 1984, 106, 4049.

[4] Y. Zhao, D. G. Truhlar, J. Chem. Theor. Comput. 2005, 1, 415.

[5] C. J. Cramer, D. G. Truhlar, Phys. Chem. Chem. Phys. 2009, 11, 10757.

[6] A. T. Bell, M. Head-Gordon, Annu. Rev. Chem. Biomol. Eng. 2011, 2, 453.

[7] F. J. Keil, Top. Curr. Chem. 2012, 307, 69.

[8] C. J. Cerjan, W. H. Miller, J. Chem. Phys. 1981, 75, 2800.

[9] J. Simons, P. Jorgensen, H. Taylor, J. Ozment, J. Phys. Chem. 1983, 87, 2745.

[10] E. Canceś, F. Legoll, M. C. Marinica, K. Minoukadeh, F. Willaime, J. Chem. Phys. 2009, 130, 114711.

[11] D. Poppinger, Chem. Phys. Lett. 1975, 35, 550.

[12] B. Peters, W.-Z. Liang, A. T. Bell, A. Chakraborty, J. Chem. Phys. 2003, $118,9533$.

[13] M. Laio, M. Parrinello, Proc. Natl. Acad. Sci. 2002, 99, 12562.

[14] M. lanuzzi, A. Laio, M. Parrinello, Phys. Rev. Lett. 2003, 90, 238302.

[15] B. Ensing, M. de Vivo, Z. Liu, P. Moore, M. L. Klein, Acc. Chem. Res. 2006, 39, 73.

[16] E. M. Mueller, A. de Meijere, H. Grubmueller, J. Chem. Phys. 2002, 116, 897.

[17] A. Samanta, E. Weinan, J. Chem. Phys. 2012, 136, 124104.

[18] S. K. Burger, P. W. Ayers, J. Chem. Phys. 2010, 132, 234110.

[19] M. Chen, M. A. Cuendet, M. E. Tuckerman, J. Chem. Phys. 2012, 137 24102.

[20] S. Maeda, K. Ohno, K. Morokuma, J. Chem. Theory Comput. 2009, 5, 2734.

[21] S. Maeda, K. Morokuma, J. Chem. Theor. Comput. 2011, 7, 2335.

[22] S. Maeda, E. Abe, M. Hatanka, T. Taketsugu, K. Morokuma, J. Chem. Theor. Comput. 2012, 8, 5058.

[23] H. B. Schlegel, J. Comput. Chem. 1982, 3, 214.

[24] G. Henkelman, H. Jonsson, J. Chem. Phys. 1999, 111, 7010.

[25] A. Heyden, A. T. Bell, F. J. Keil, J. Chem. Phys. 2005, 123, 224101.

[26] J. Baker, J. Comput. Chem. 1986, 7, 385.

[27] D. J. Wales, J. Chem. Soc. Faraday Trans. 1992, 88, 653.

[28] J. M. del Campo, A. M. Koster, J. Chem. Phys. 2008, 129, 024107.

[29] H. B. Schlegel, WIREs Comput. Mol. Sci. 2011, 1, 790.

[30] H. B. Schlegel, J. Comput. Chem. 2003, 24, 1514.

[31] J. W. May, J. D. Lehner, M. J. Frisch, X. S. Li, J. Chem. Theor. Comput. 2012, 8, 5175 .

[32] X. S. Li, M. J. Frisch, J. Chem. Theor. Comput. 2006, 2, 835.

[33] W. Liang, H. Wang, J. Hung, X. S. Li, M. J. Frisch, J. Chem Theor. Comput. 2010, 6, 2034.

[34] J. Baker, A. Kessi, B. Delley, J. Chem. Phys. 1996, 105, 192.

[35] V. Bakken, T. Helgaker, J. Chem. Phys. 2002, 117, 9160.

[36] S. R. Billeter, A. J. Turner, W. Thiel, Phys. Chem. Chem. Phys. 2000, 2, 2177.

[37] J. Baker, J. Comput. Chem. 1993, 14, 1085.

[38] P. Pulay, G. Fogarasi, J. Chem. Phys. 1992, 96, 2856.

[39] B. Paizs, J. Baker, S. Suhai, P. Pulay, J. Chem. Phys. 2000, 133, 6566.

[40] A. Banerjee, N. Adams, J. Simons, R. Shepard, J. Phys. Chem. 1995, 89, 52.

[41] J. Baker, F. Chan, J. Comput. Chem. 1996, 17, 888.

[42] R. Granot, B. A. Baer, J. Chem. Phys. 2008, 128, 184111.

[43] S. A. Ghasemi, S. Goedecker, J. Chem. Phys. 2011, 135, 014108.

[44] P. Y. Ayala, H. B. Schlegel, J. Chem. Phys. 1997, 107, 375.

[45] C. Peng, P. Y. Ayala, H. B. Schlegel, M. J. Frisch, J. Comput. Chem. 1996, 17, 49.

[46] C. Peng, H. B. Schlegel, Israel J. Chem. 1994, 33, 449.

[47] G. Mills, H. Jonsson, Phys. Rev. Lett. 1994, 72, 1124.

[48] G. Henkelman, H. Jonsson, J. Chem. Phys. 2000, 113, 9978

[49] G. Henkelman, B. P. Uberuaga, H. J. Jonsson, J. Chem. Phys. 2000, 113,9901

[50] S. A. Trygubenko, D. J. Wales, J. Chem. Phys. 2004, 120, 2082.

[51] J. Chu, B. Trout, B. A. Brooks, J. Chem. Phys. 2003, 119, 12708.
[52] D. Sheppard, R. Terrell, G. Henkelman, J. Chem. Phys. 2008, 128, 134106.

[53] W. E, W. Ren, E. Vanden-Eijnden, Phys. Rev. B 2002, 66, 052301.

[54] E. Weinan, W. Ren, E. Vanden-Eijnden, J. Phys. Chem. B 2005, 109, 6688.

[55] W. Ren, E. Vanden-Eijnden, J. Chem. Phys. 2007, 126, 164103.

[56] S. K. Burger, W. Yang, J. Chem. Phys. 2006, 24, 054109.

[57] S. K. Burger, W. Yang, J. Chem. Phys. 2007, 127, 164107.

[58] H. Chaffey-Millar, A. Nikodem, A. V. Matveev, S. Krüger, N. Rösch, J. Chem. Theor. Comput. 2012, 8, 777.

[59] J. Jung, S. Re, S. Ten-no, J. Chem. Phys. 2013, 138, 044106.

[60] Y. Liu, S. K. Burger, P. W. Ayers, J. Math. Chem. 2011, 49, 1915.

[61] P. Tao, M. Hodoscek, J. D. Larkin, Y. Shao, B. R. Brooks, J. Chem. Theor. Comput. 2012, 8, 5035.

[62] J. Klimes, D. R. Bowler, A. Michaelides, J. Phys. Condens. Matter 2010, 22, 074203.

[63] P. Plessow, J. Chem. Theor. Comput. 2013, 9, 1305.

[64] W. Ren, E. Vanden-Eijnden, J. Chem. Phys. 2013, 138, 134105.

[65] B. Peters, A. Heyden, A. T. Bell, A. Chakraborty, J. Chem. Phys. 2004, 120, 7877.

[66] A. Goodrow, A. T. Bell, M. Head-Gordon, J. Chem. Phys. 2008, 129, 174109.

[67] W. A. Quapp, J. Chem. Phys. 2005, 122, 174106.

[68] A. Behn, P. M. Zimmerman, A. T. Bell, M. Head-Gordon, J. Chem. Theor. Comput. 2011, 7, 4019.

[69] S. M. Sharada, P. M. Zimmerman, A. T. Bell, M. Head-Gordon, J. Chem. Theor. Comput. 2012, 8, 5166.

[70] A. Behn, P. M. Zimmerman, A. T. Bell, M. Head-Gordon, J. Chem. Phys. 2011, 135, 224108.

[71] P. M. Zimmerman, J. Chem. Phys. 2013, 138, 184102.

[72] P. M. Zimmerman, J. Chem. Theor. Comput. 2013, 9, 3043.

[73] J. M. Anglada, E. Besalu, J. M. Bofill, R. Creheut, J. Comput. Chem. 2001, 22, 387.

[74] J. M. Bofill, J. M. Anglada, Theor. Chem. Acc. 2001, 105, 463.

[75] T. Lankau, C.-H. Yu, J. Chem. Phys. 2013, 138, 214102.

[76] W. Quapp, M. Hirsch, O. Imig, D. Heidrich, J. Comput. Chem. 1998, 19, 1087.

[77] J. M. Bofill, W. Quapp, J. Chem. Phys. 2011, 134, 074101.

[78] Y. Liu, S. K. Burger, P. W. Ayers, J. Math. Chem. 2011, 49, 1915.

[79] S. K. Burger, P. W. Ayers, J. Chem. Phys. 2010, 132, 234110.

[80] C. Sheng, Z.-P. Liu, J. Chem. Theor. Comput. 2012, 8, 2215.

[81] C. G. Broyden, J. Inst. Math. Appl. 1970, 6, 76.

[82] R. Fletcher, Comput. J. 1970, 13, 317.

[83] D. Goldfarb, Math. Comput. 1970, 24, 23.

[84] D. F. Shanno, Math. Comput. 1970, 24, 647.

[85] J. M. Bofill, J. Comput. Chem. 1994, 15, 1.

[86] P. M. Zimmerman, J. Comput. Chem. 2013, 34, 1385.

[87] P. M. Zimmerman, Mol. Simul. 2015, 41, 43-54.

[88] E. V. Anoshkina, A. G. Belyaev, H.-P. Seidel, In Proceedings of Vision, Modeling, and Visualization (VMV); Erlangen, Germany, 2002.

[89] S. M. Sharada, A. T. Bell, M. Head-Gordon, J. Chem. Phys. 2014, 140, 164115.

[90] E. R. Davidson, J. Comput. Phys. 1975, 17, 87.

[91] A. D. Becke, Phys. Rev. A 1998, 38, 3098.

[92] C. Lee, W. Yang, R. G. Parr, Phys. Rev. B 1988, 37, 785.

[93] A. D. Becke, J. Chem. Phys. 1993, 98, 5648.

[94] P. J. Hay, W. R. Wadt, J. Chem. Phys. 1985, 82, 270.

[95] P. J. Hay, W. R. Wadt, J. Chem. Phys. 1985, 82, 284.

[96] P. J. Hay W. R. Wadt, J. Chem. Phys. 1985, 82, 299.

[97] Y. Shao, L. Fusti-Molnar, Y. Jung, J. Kussmann, C. Ochsenfeld, S. T. Brown, A. T. B. Gilbert, L. V. Slipchenko, S. V. Levchenko, D. P. O'Neill, R. A. Distasio Jr., R. C. Lochan, T. Wang, G. J. O. Beran, N. A. Besley, J. M., Herbert, C. Y. Lin, T. Van Voorhis, S. H. Chien, A. Sodt, R. P. Steele, V. A. Rassolov, P. E. Maslen, P. P. Korambath, R. D. Adamson, B. Austin, J. Baker, E. F. C. Byrd, H. Dachsel, R. J. Doerksen, A. Dreuw, B. D. Dunietz, A. D. Dutoi, T. R. Furlani, S. R. Gwaltney, A. Heyden, S. Hirata, C.-P. Hsu, G. Kedziora, R. Z. Khalliulin, P. Klunzinger, A. M. Lee, M. S. Lee, W. Liang, I. Lotan, N. Nair, B. Peters, E. I. Proynov, P. A. Pieniazek, Y. M. Rhee, J. Ritchie, E. Rosta, C. D. Sherrill, A. C. Simmonett, J. E. Subotnik, H. L. Woodcock III, W. Zhang, A. T. Bell, A. K. Chakraborty, D. M. Chipman, F. J. Keil, A. Warshel, W. J. Hehre, H. F. Schaefer III, J. 
Kong, A. I. Krylov, P. M. W. Gill, M. Head-Gordon, Phys. Chem. Chem. Phys. 2006, 8, 3172.

[98] W. Humphrey, A. Dalke, K. Schulten, J. Mol. Graphics 1996, 14, 33.

[99] O. Diels, L. Alder, Liebigs Ann. Chem. 1928, 460, 98.

[100] A. Perczel, O. Farkas, I. Jakli, I. A. Topol, I. G. Csizmadia, J. Comput. Chem. 2003, 24, 1026.

[101] A. E. Shilov, G. B. Shul'pin, Chem. Rev. 1997, 97, 2879.

[102] M. Lersch, M. Tilset, Chem. Rev. 2005, 105, 2471.

[103] A. Paul, C. B. Musgrave, Organometallics 2007, 26, 793.

[104] J. R. Locke, A. J. McNeil, Macromolecules 2010, 43, 8709.

[105] Z. J. Bryan, A. J. McNeil, Chem. Sci. 2013, 4, 1620.

[106] A. Paul, C. B. Musgrave, Angew. Chem. Int. Ed. 2007, 46, 8153.

[107] J. M. Rose, F. Deplace, N. A. Lynd, Z. Wang, A. Hotta, E. B. Lobkovsky, E. J. Kramer, G. W. Coates, Macromolecules 2008, 41, 9548.

[108] J. M. Rose, A. E. Cherian, G. W. Coates, J. Am. Chem. Soc. 2006, 128, 4186.
[109] A. D. Ryabov, A. K. Sakodinskaya, A. K. Yatsimirsky, J. Chem. Soc. Dalton Trans. 1985, 1985, 2629.

[110] M. Lafrance, S. I. Gorelsky, K. Fagnou, J. Am. Chem. Soc. 2007, 129, 14570.

[111] A. Maleckis, J. W. Kampf, M. S. Sanford, J. Am. Chem. Soc. 2013, 135, 6618.

[112] T. B. Lee, M. L. McKee, Inorg. Chem. 2009, 48, 7564.

[113] Z. Xiong, C. K. Yong, G. Wu, P. Chen, W. Shaw, A. Karkamkar, T. Autrey, M. O. Jones, S. R. Johnson, P. P. Edwards, W. I. F. David, Nat. Mater. 2008, 7, 138.

Received: 9 November 2014

Accepted: 14 December 2014

Published online on 8 January 2015 